\title{
Prevalencia del uso de drogas ilegales en función del consumo de tabaco en una muestra de estudiantes en México
}

\author{
Luisa María Sánchez-Zamorano, Dra en C, (') Angélica Ángeles Llerenas, M en C, (I) Rafael Anaya-Ocampo, M en C, (I)
} Eduardo Lazcano-Ponce, Dr en C. ${ }^{(1)}$

\begin{abstract}
Sánchez-Zamorano LM, Ángeles Llerenas A, Anaya-Ocampo R, Lazcano-Ponce E. Prevalencia del uso de drogas ilegales en función del consumo de tabaco en una muestra de estudiantes en México. Salud Publica Mex 2007;49 supl 2:SI 82-SI 93.
\end{abstract}

\section{Resumen}

Objetivo. Evaluar el uso de drogas ilegales y su interacción con el consumo de tabaco en jóvenes estudiantes de un estado de la región central de México, 2005-2007. Material y métodos. Se realizó un estudio de cohorte en el ámbito escolar en el estado de Morelos. La población fue de 4625 alumnos. Se efectuó el análisis de la medición basal describiendo el consumo de tabaco, alcohol y drogas ilegales; se evaluó la diferencia del consumo de estas últimas mediante modelos de regresión logística y se estratificó por el antecedente de fumar. Resultados. El promedio de edad fue I3.8 (DE 2.03). La proporción de mujeres fue de $51.9 \%$; II.9\% notificó el antecedente de consumo de tabaco (13.5\% hombres y $10.4 \%$ mujeres). El uso de drogas ilegales fue de 3.3\% (3.7\% hombres y $2.8 \%$ mujeres), su distribución según el consumo de tabaco fue de $2.0 \%$ en los no fumadores y $12.4 \%$ en los que tenían antecedente de consumo de tabaco. En el grupo de no fumadores, el consumo de drogas ilegales por parte de los padres incrementó 4.5 veces (IC95\% I.9-I I.0) la posibilidad de que un adolescente usara drogas ilegales en relación con aquellos cuyos padres no consumen drogas ilegales, relación no significativa en el grupo de fumadores. Para este último grupo, el consumo de alcohol se asoció con el uso de drogas ilegales $(O R=2.6$; IC95\% I.2-5.9). Conclusiones. El control

\author{
Sánchez-Zamorano LM, Ángeles Llerenas A, \\ Anaya-Ocampo R, Lazcano-Ponce E. \\ Prevalence of illicit use in function of tobacco smoking in \\ Mexican students sample. \\ Salud Publica Mex 2007;49 suppl 2:SI82-SI 93.
}

\section{Abstract}

Objective. To evaluate the use of the illicit drugs and its interaction with smoking in young students in a state of Central Mexico, 2005-2007. Material and Methods. A population cohort study with 4625 students was carried out in different public schools in Morelos State. Baseline measurement analysis was carried out describing tobacco, alcohol and illegal drugs consumption. Drug abuse according to tobacco onsumption was assessed through logistic regression models, stratified by smoking habit. Results. Mean age was 13.8 (SD 2.03); $51.9 \%$ were female students and $11.9 \%$ of total population informed having smoked (13.5\% men and $10.4 \%$ women). Illicit drug use was $3.3 \% ; 3.7 \%$ in men and $2.8 \%$ in women and its distribution according tobacco smoking was $2.0 \%$ for non smokers and $12.4 \%$ for those who had smoked. In non smokers group, parent's drug use was 4.5 times (C195\% I.9-I I.0) higher compared to those students whose parents do not do drug use. The effect was not significant in smokers group.Alcohol intake was associated to drug abuse in smokers group $(O R=2.5 ; C 195 \%$ I.2-5.9). Conclusions. The control and prevention of tobacco consumption and other addictive substances should be carried out since in a comprehensive way, because contextual antecedent these behaviors are related.

(I) Instituto Nacional de Salud Pública, México.

Fecha de recibido: 4 de abril de 2007 - Fecha de aceptado: 30 de abril de 2007 Solicitud de sobretiros: Dra. Luisa María Sánchez-Zamorano, Dirección de Evaluación de Programas y Bioestadística,

Centro de Investigación en Salud Poblacional, Instituto Nacional de Salud Pública,

Av. Universidad 655, Col. Santa María Ahuacatitlán 62508, Cuernavaca, Mor., México. Email: szamoran@insp.mx 
y prevención del consumo de tabaco y otras sustancias adictivas debe realizarse de manera integral, ya que vistas en su contexto tales conductas se encuentran relacionadas.

Palabras clave: adolescentes; tabaco; drogas ilegales; cohorte; México
Key words: adolescents; tobacco; illicit drugs; cohort; Mexico
E la actualidad, la prevención de los adolescentes ante el inicio temprano en el consumo de tabaco, as como de drogas ilegales y de la ingesta inmoderada de alcohol, se ha convertido en una prioridad en materia de salud pública. En los pa ses desarrollados, el uso de drogas ilegales entre los adolescentes se incrementó desde 1990. Según datos obtenidos de encuestas realizadas en escolares, la marihuana es la droga il cita de mayor consumo en todo el mundo; acompaña al consumo inmoderado de alcohol y en general es más frecuente entre los hombres. ${ }^{1-5}$ En México, el uso de sustancias adictivas entre la población estudiantil se ha documentado mediante la aplicación, en tres ocasiones (1976, 1986 y 1991), de la Encuesta Nacional de Uso de Drogas entre la Comunidad Escolar (ENUDCE), as como de estudios espec ficos en escolares de la Ciudad de México que se realizan con una periodicidad de dos o tres años. ${ }^{6}$ En Morelos, en 1998, se notificó una prevalencia de $5.8 \%$ en el uso de drogas ilegales en estudiantes de 11 a 24 años de edad, mayor en hombres (9.1\%) que en mujeres $(3.3 \%){ }^{7}$

En lo tocante al consumo de tabaco, en México se han desarrollado estudios epidemiológicos cuyo propósito ha sido evidenciar el problema del tabaquismo en la población general; algunos de esos trabajos se han enfocado en los adolescentes. La última Encuesta Nacional de Adicciones (ENA), aplicada en 2002, indicó que la prevalencia de consumo de tabaco en jóvenes de 12 a 17 años fue de $15.4 \%$ en hombres y $4.8 \%$ en mujeres. ${ }^{8}$ La Encuesta sobre Tabaquismo en Jóvenes (GYTS, por sus siglas en inglés), aplicada en áreas urbanas, evidenció cifras alarmantes. En general, la prevalencia de adolescentes de 12 a 15 años de edad que hab an fumado en el último mes previo a la encuesta fue de $20.0 \%$. Al estratificar por sexo, la prevalencia en varones fue de $20.8 \%$ y en mujeres de $18.2 \%$. En la Ciudad de Cuernavaca, Morelos, la prevalencia fue menor en hombres $(17.6 \%)$ respecto de las mujeres $(22.9 \%) .{ }^{9}$ Finalmente, en la Encuesta Nacional de Salud y Nutrición 2006 (ENSANUT 2006) las prevalencias de consumo de tabaco por sexo fueron menores en los adolescentes de 13 a 15 años de edad (4.6\% para los hombres vs. $2.4 \%$ en las mujeres). ${ }^{10}$
Respecto al consumo de alcohol, la ENSANUT 2006 informó una prevalencia de $17.7 \%$ en adolescentes; las cifras más altas $(21.4 \%)$ correspondieron a los varones y las más bajas (13.9\%) a las mujeres. En el estudio realizado en 1998 en la población del estado de Morelos, se menciona que la prevalencia del antecedente de consumo de alcohol en estudiantes de 11 a 24 años de edad fue de $35.2 \%$; ésta fue mayor en hombres (40\%) que en mujeres $(31.4 \%){ }^{7}$

La relación entre el consumo de tabaco y el uso de otras sustancias adictivas, como las drogas ilegales y el alcohol, se ha explicado mediante modelos teóricos, entre los que destacan el de la "Piedra angular" o el de la "Puerta de entrada", 11-15 as como la "Teor a del problema de conducta", que se basa en el ambiente social, en la percepción del ambiente que tiene el individuo y en la personalidad de este último. Lo cierto es que, en general, estos modelos son multifactoriales y a menudo se combinan sistemas psicosociales y otras conductas que, en el caso del uso de drogas ilegales, representan una constelación o s ndrome que incluye varias formas de abuso de sustancias (tabaco, alcohol y drogas ilegales), al igual que otras conductas negativas como mentir o robar. ${ }^{16}$

Ahora bien, se considera que la niñez y la adolescencia son etapas de la vida de alto riesgo con respecto al consumo de tabaco, a la ingesta inmoderada de alcohol y al uso de drogas ilegales, pues es en dichas fases del desarrollo cuando los individuos se inician en su consumo experimental. ${ }^{17-19}$ En la adolescencia se transita por un proceso de autodefinición personal que, aunado a las estructuras intelectuales y caracter sticas socioafectivas de esta población, ${ }^{20}$ ponen a los jóvenes en riesgo de empezar a manejar las tensiones a través del consumo de tóxicos. ${ }^{17-19}$ Asimismo, de acuerdo con el tipo de sustancia, el efecto sobre la salud puede ser de corto, mediano o largo plazo. En el caso de las drogas ilegales, se pueden observar consecuencias adversas en el corto plazo que incluyen asociaciones con otros riesgos psicosociales como accidentes y violencia, embarazo no planeado e infecciones de transmisión sexual, entre otros. ${ }^{2}$ Desde esta perspectiva, los estilos de vida que vulneran el 
desarrollo pleno de la niñez y la adolescencia constituyen el blanco de la prevención. ${ }^{21}$

Tomando en cuenta lo anterior y en el interés de destacar el papel que desempeña el consumo de tabaco con respecto al uso de drogas ilegales, en este estudio se buscó realizar el diagnóstico del uso de drogas ilegales y evaluar su relación con el antecedente de consumo de tabaco en combinación con el consumo de alcohol, as como las variables socioeconómicas y personales en jóvenes estudiantes de un estado de la región central de México.

\section{Material y métodos}

\section{Población de estudio}

La información del presente trabajo procede de la medición basal de un estudio de cohorte realizado en estudiantes de escuelas públicas del estado de Morelos, México. La muestra original se conformó con 4625 jóvenes de escuelas secundarias, preparatorias y profesionales que aceptaron participar en el mismo. La tasa de participación fue de $92.5 \%$ entre los estudiantes seleccionados a partir de un muestreo aleatorio de las escuelas del sistema público de educación de la entidad, donde se entrevistó exclusivamente a alumnos del primer año de cada nivel educativo durante el periodo escolar 20052006. La muestra total de estudiantes se distribuyó en 11 escuelas de grado medio (secundarias), cinco de grado medio superior (bachilleratos) y tres de grado profesional. El comité de ética del Instituto Nacional de Salud Pública aprobó el presente proyecto, que también se presentó ante las autoridades y padres de familia de las escuelas. En el caso de los participantes menores de 18 años, se aceptó la participación en el estudio de aquellos estudiantes que aceptaron participar y cuyos padres y/o tutores otorgaron el correspondiente consentimiento informado mediante la firma de una carta.

\section{Variable dependiente}

Con las siguientes preguntas: “ ¿Fumas, tomas, te inyectas o inhalas algún tipo de droga aparte del cigarro?" y “¿Has probado alguna vez drogas ilegales?”, se construyó la variable dependiente. Ambas se relacionan con el consumo actual o previo de drogas ilegales (coca na, marihuana, hero na, cemento, thinner, crack, morfina, chochos, éxtasis, anfetaminas, hongos, polvo de ángel, puc/ pus, tachas). La variable se conformó con aquellos estudiantes que indicaron no tener ningún antecedente de consumo de alguna de esas sustancias (categor a de referencia) y con aquellos que reconocieron haber consumido alguna vez y/o consumir en la actualidad alguna de las drogas ilegales antes mencionadas.

\section{Variables independientes}

La variable independiente de interés fue el antecedente de consumo de tabaco, con dos categor as: la de no exposición, que comprendió a los que respondieron que no fumaban, y la del antecedente de consumo de tabaco, integrada por los jóvenes que indicaron fumar actualmente y por aquellos que afirmaron que antes lo hac an pero que en el presente no fumaban. Asimismo, se incluyeron las siguientes variables: a) sociodemográficas: sexo, edad en años cumplidos, grado de educación, ingreso familiar y estado civil de los padres; b) adicciones: antecedente de consumo de alcohol, uso de drogas ilegales por parte de los padres y amigos, antecedente de que le hayan ofrecido drogas ilegales y lugar donde eso ocurrió, as como información recibida de los padres y profesores acerca del peligro que representan las drogas; y c) psicológicas: sintomatolog a depresiva, construida a partir de la escala de Zung ${ }^{22}$ categorizada de acuerdo con los criterios de Seva-Diaz ${ }^{23}$ en los niveles de ausencia de depresión, depresión leve y depresión moderada-severa; después se dicotomizó agrupando las últimas dos categor as. Otras variables de interés fueron la actividad deportiva de los estudiantes, as como aquellas variables relacionadas con su perspectiva acerca de su relación familiar, a partir de las siguientes preguntas:" ¿Estás satisfecho con la ayuda que recibes de tu familia?"; "En tu familia ¿conversan entre ustedes los problemas que tienen en casa?"; “¿Las decisiones importantes se toman en conjunto en casa?"; “ ¿Estás satisfecho con el tiempo que tu familia y tú pasan juntos? "; y, por último, "¿Sientes que tu familia te quiere?"

\section{Análisis estadístico}

El análisis estad stico se realizó en tres etapas: la primera consistió en un análisis descriptivo para observar la distribución del uso de drogas ilegales de acuerdo con las variables sociodemográficas, los antecedentes familiares, el antecedente de uso de drogas ilegales en la familia y los pares, el hecho de haber estado expuesto al ofrecimiento de drogas ilegales y el lugar donde ocurrió, y las drogas notificadas que han consumido, con estratificación por el antecedente de consumo de tabaco. Para controlar la confusión residual ocasionada por variables que explican el consumo de tabaco en los jóvenes y que podr an relacionarse con el consumo de drogas ilegales, se optó por construir un " ndice de propensidad" a partir de las probabilidades predichas para que un estudiante presente el antecedente de fumar, mediante la construcción de un modelo de regresión log stica múltiple en el que se utilizaron las siguientes variables explicativas para el antecedente de consumo 
de tabaco en los jóvenes: a) consumo de tabaco por parte de los padres, el mejor amigo, el hermano, la pareja, algún compañero de clase, algún profesor; b) la presencia de letreros en la escuela que proh ban fumar y conocimiento de que el tabaco es una droga. Más adelante, se incluyó este ndice en los modelos que se construyeron para explicar la relación de las variables de interés con el antecedente de consumo de drogas ilegales, con estratificación por antecedente de consumo de tabaco.

Más tarde se realizaron modelos de regresión log stica múltiple utilizando la modalidad de efectos mixtos (multinivel) a fin de evaluar si la escuela, como variable de agrupamiento, explicaba significativamente algún porcentaje de la varianza, donde la variable dependiente fue el uso de drogas ilegales. Al no resultar as, se utilizaron modelos de regresión log stica múltiple donde se muestran las variables que explicaban el uso de drogas ilegales y las diferencias de acuerdo con la condición de haber fumado alguna vez, cada modelo ajustado por el ndice de propensidad referido. Por último, en cada modelo final se evaluó la bondad de ajuste mediante la prueba de Hosmer-Lemeshow. Se utilizó el paquete estad stico STATA ver $9.0^{24}$ para llevar a cabo los análisis.

\section{Resultados}

\section{Generalidades}

La población de estudio fue de 4625 estudiantes y el promedio de edad de 13.84 años (DE 2.03); su distribución por escolaridad se muestra en la figura 1. De dicha población, $51.9 \%$ era mujer; de $11.9 \%$ de jóvenes que informaron el antecedente de consumo de tabaco, $13.5 \%$ era varón y $10.4 \%$ mujer. Con respecto al uso de drogas ilegales, la cifra ascendió a 3.3\%, de la cual 3.7\% era hombre y $2.8 \%$ mujer y, de acuerdo con el antecedente de consumo de tabaco, fue de $2.0 \%$ para los que no fumaban y de $12.4 \%$ para los que ten an antecedente de haber fumado. En lo tocante al tipo de droga consumida, la distribución fue diferente entre los fumadores y los no fumadores. Se observaron las siguientes prevalencias de inhalables en los no fumadores: thinner $(19.3 \%)$ y cemento (18.1\%); éstas fueron las drogas de mayor uso. Por otra parte, en los fumadores la coca na fue la principal droga consumida (47.1\%), seguida de cemento $(33.8 \%)$ y de marihuana $(33.8 \%)$, ya sea en la modalidad de uso único o de policonsumo (cuadro I).

\section{Uso de drogas ilegales}

La distribución del uso de drogas ilegales según las variables de interés, estratificada por antecedente de consumo de tabaco, se muestra en el cuadro II, donde puede observarse una relación estad sticamente significativa entre el consumo de drogas y el antecedente de fumar, que se da en las variables relacionadas con la percepción del estudiante de su relación familiar. Por otra parte, en el cuadro III se presenta la distribución por antecedentes familiares y de pares de uso de drogas ilegales, as como información sobre dichas sustancias y el hecho de estar expuesto al ofrecimiento de las mismas en diferentes lugares. Asimismo, se aprecia una mayor prevalencia del uso de drogas ilegales de los estudiantes, en el caso de que los padres y los amigos consuman drogas, de que no hayan tenido información sobre el riesgo de consumir drogas y de que se las hayan ofrecido en algún momento, siendo mayores, en general, las prevalencias en el grupo de fumadores respecto de los no fumadores.

\section{Drogas ilegales y consumo de tabaco}

La distribución del uso de drogas ilegales de acuerdo con el consumo de tabaco y/o alcohol se muestra en la figura 2; en aquellos estudiantes que tuvieron antecedentes de consumo de tabaco, la prevalencia de uso de drogas ilegales fue mayor que en el caso de los que ten an antecedentes de consumo de alcohol $(6.37 \%$ vs. 3.01\%). La prevalencia más elevada se presenta en aquellos que notificaron el consumo conjunto de tabaco y alcohol (15.9\%).

\section{Factores asociados al consumo de drogas ilegales}

Las caracter sticas que se observaron y que explican el uso de drogas ilegales de acuerdo con el antecedente de consumo de tabaco se muestran en el cuadro IV, donde se puede apreciar que, en los no fumadores, el efecto que tiene el uso de drogas ilegales por parte de alguno de los padres es 4.5 veces mayor (IC95\% 1.9-11.0) en comparación con aquellos jóvenes cuyos padres no consumen drogas ilegales; este efecto no fue significativo en el grupo de estudiantes con antecedente de fumar. Para este último grupo, el consumo de alcohol se asoció positivamente con el uso de drogas ilegales $(O R=$ 2.6; IC95\% 1.2-5.9); sin embargo, en el grupo de los no fumadores no se observó una relación estad sticamente significativa.

En cuanto a las variables de percepción de la relación con la familia, sólo la relativa a si los estudiantes sienten que su familia los quiere mostró estar relacionada para el grupo que ten a antecedente de fumar; espec ficamente, para la categor a de aquellos que contestaron "casi nunca", en comparación con los que 


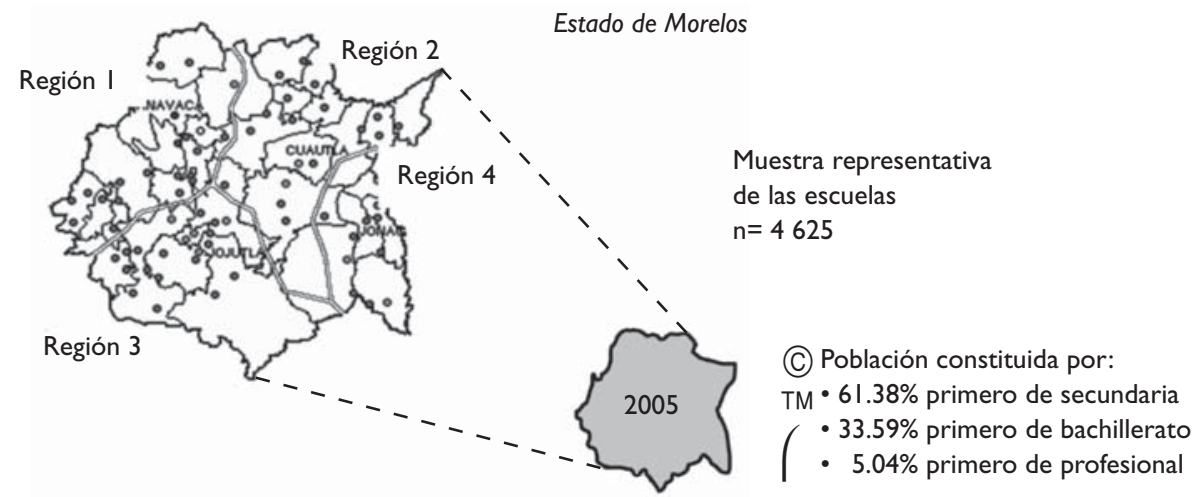

Figura I. Diseño del estudio basal de estudiantes del Estado de Morelos, México, 2005

Cuadro I

Prevalencia y distribución porcentual del uso de dRogas ilegales en función del Consumo de tabaco y género en estudiantes, Morelos, México, 2005

\begin{tabular}{|c|c|c|c|c|c|c|}
\hline \multirow[b]{2}{*}{ Sustancia } & \multicolumn{2}{|c|}{$\begin{array}{c}\text { Total } \\
n=I 5 I(3.3)^{*}\end{array}$} & \multicolumn{2}{|c|}{$\begin{array}{c}\text { No fumadores } \\
n=83(2.0)\end{array}$} & \multicolumn{2}{|c|}{$\begin{array}{c}\text { Fumadores } \\
n=68(12.4)\end{array}$} \\
\hline & $n$ & $\%$ & $n$ & $\%$ & $n$ & $\%$ \\
\hline Cocaína & 43 & 28.48 & II & 13.25 & 32 & 47.06 \\
\hline Cemento & 38 & 25.17 & 15 & 18.07 & 23 & 33.82 \\
\hline Marihuana & 28 & 18.54 & 10 & 12.05 & 18 & 26.47 \\
\hline Thinner & 28 & 18.54 & 16 & 19.28 & 12 & 17.65 \\
\hline Crack & 9 & 5.96 & 8 & 9.64 & I & 1.47 \\
\hline Éxtasis & 9 & 5.96 & 3 & 3.61 & 6 & 8.82 \\
\hline Heroína & 8 & 5.30 & 4 & 4.82 & 4 & 5.88 \\
\hline Morfina & 8 & 5.30 & 6 & 7.23 & 2 & 2.94 \\
\hline Hongos & 7 & 4.64 & 5 & 6.02 & 2 & 2.94 \\
\hline Esteroides & 7 & 4.64 & 5 & 6.02 & 2 & 2.94 \\
\hline Anfetaminas & 6 & 3.97 & 5 & 6.02 & I & 1.47 \\
\hline Polvo de ángel & 6 & 3.97 & 4 & 4.82 & 2 & 2.94 \\
\hline Puc/Pus & 6 & 3.97 & 5 & 6.02 & I & 1.47 \\
\hline Tachas & 5 & 3.31 & 3 & 3.61 & 2 & 2.94 \\
\hline
\end{tabular}

\begin{tabular}{|c|c|c|c|c|}
\hline \multirow[b]{2}{*}{ Sustancia } & \multicolumn{2}{|c|}{$\begin{array}{c}\text { Mujeres } \\
n=68(2.8)\end{array}$} & \multicolumn{2}{|c|}{$\begin{array}{c}\text { Hombres } \\
n=83(3.7)\end{array}$} \\
\hline & $n$ & $\%$ & $n$ & $\%$ \\
\hline Cocaína & 14 & 20.59 & 29 & 34.94 \\
\hline Cemento & 10 & $14.7 \mid$ & 28 & 33.73 \\
\hline Marihuana & 17 & 25.00 & $\mathrm{II}$ & 13.25 \\
\hline Thinner & 10 & $|4.7|$ & 18 & 21.69 \\
\hline Crack & 1 & 1.47 & 8 & 9.64 \\
\hline Éxtasis & 3 & $4.4 I$ & 6 & 7.23 \\
\hline Heroína & 3 & $4.4 I$ & 5 & 6.02 \\
\hline Morfina & 3 & $4.4 I$ & 5 & 6.02 \\
\hline Hongos & 0 & 0.00 & 7 & 8.43 \\
\hline Esteroides & I & 1.47 & 6 & 7.23 \\
\hline Anfetaminas & I & $\mathrm{I} .47$ & 5 & 6.02 \\
\hline Polvo de ángel & I & 1.47 & 5 & 6.02 \\
\hline Puc/Pus & 0 & 0.00 & 6 & 7.23 \\
\hline Tachas & 0 & 0.00 & 5 & 6.02 \\
\hline
\end{tabular}




\section{Cuadro II}

Prevalencia del uso de drogas ilegales en función del antecedente de tabaquismo y características SOCIOECONómicas Y PSICOSOCIALES De MoRelos, MéXıCO, 2005

\begin{tabular}{|c|c|c|c|c|c|}
\hline \multirow[b]{2}{*}{ Variable } & \multicolumn{2}{|c|}{ No fumadores } & \multicolumn{3}{|c|}{ Fumadores } \\
\hline & $n$ & \%* & $n$ & $\% *$ & Valor " $p$ ": \\
\hline \multicolumn{6}{|l|}{ Características sociodemográficas } \\
\hline \multicolumn{6}{|l|}{ Género } \\
\hline Mujeres & 2148 & 1.82 & 250 & 11.60 & 0.000 \\
\hline Hombres & 1927 & 2.28 & 300 & 13.00 & 0.000 \\
\hline \multicolumn{6}{|l|}{ Edad (años) } \\
\hline 11 a 12 & 1419 & 2.18 & 49 & 12.24 & 0.000 \\
\hline 13 a 15 & 2110 & 1.75 & 276 & 9.42 & 0.000 \\
\hline 16 a 17 & 364 & 3.02 & 129 & 14.73 & 0.000 \\
\hline 18 o más & 182 & 2.20 & 96 & $17.7 \mid$ & 0.000 \\
\hline \multicolumn{6}{|l|}{ Ingreso familiar } \\
\hline$\leq \$ 600.00$ & 1513 & 2.11 & 145 & 9.66 & 0.000 \\
\hline$\$ 601.00$ a $\$ 1,200.00$ & 1321 & 1.59 & 220 & 15.00 & 0.000 \\
\hline$\$ 1,201.00$ a $\$ 2,400.00$ & 676 & 1.78 & 122 & 11.48 & 0.000 \\
\hline$>\$ 2,400.00$ & 565 & 2.12 & 63 & II.II & 0.000 \\
\hline \multicolumn{6}{|l|}{ Escolaridad de la madre } \\
\hline Primaria & 1861 & 1.83 & 189 & 7.41 & 0.000 \\
\hline Secundaria & 1049 & 2.67 & 138 & 12.32 & 0.000 \\
\hline Preparatoria/Universidad & 1165 & 1.80 & 223 & 16.59 & 0.000 \\
\hline \multicolumn{6}{|l|}{ Escolaridad del padre } \\
\hline Primaria & 1876 & 2.24 & 214 & 10.28 & 0.000 \\
\hline Secundaria & 1040 & 1.83 & 131 & 12.98 & 0.000 \\
\hline Preparatoria/Universidad & 1159 & 1.90 & 205 & 14.15 & 0.000 \\
\hline \multicolumn{6}{|l|}{ Estado civil de los padres } \\
\hline Con pareja (casados, unión libre) & 2336 & 1.41 & 261 & 11.49 & 0.000 \\
\hline Separados (divorciados, viudos, madres solteras, separados) & 1099 & 2.64 & 208 & 14.42 & 0.000 \\
\hline
\end{tabular}

Características psicosociales

Consumo de alcohol alguna vez en la vida

\begin{tabular}{|c|c|c|c|c|c|}
\hline No & 3407 & 1.73 & 180 & 5.00 & 0.000 \\
\hline Sí & 664 & 3.01 & 346 & 15.90 & 0.000 \\
\hline \multicolumn{6}{|c|}{ Síntomas depresivos } \\
\hline Sin síntomas & 799 & 1.88 & 117 & 9.40 & 0.000 \\
\hline Con síntomas & 3276 & 2.08 & 433 & 13.16 & 0.000 \\
\hline \multicolumn{6}{|c|}{ Satisfecho con la ayuda familiar } \\
\hline Casi siempre & 2797 & 1.54 & 333 & $10.5 \mid$ & 0.000 \\
\hline A veces & 1060 & 2.45 & 182 & 12.64 & 0.000 \\
\hline Casi nunca & 218 & 6.42 & 35 & 28.57 & 0.000 \\
\hline \multicolumn{6}{|c|}{ Resolución de problemas en familia } \\
\hline Casi siempre & 1832 & 1.47 & 230 & 12.17 & 0.000 \\
\hline A veces & 1707 & 2.23 & 227 & 12.33 & 0.000 \\
\hline Casi nunca & 536 & 3.36 & 93 & 12.90 & 0.000 \\
\hline \multicolumn{6}{|c|}{ Toma de decisiones en familia } \\
\hline Casi siempre & 1885 & 1.64 & 234 & 12.82 & 0.000 \\
\hline A veces & 1693 & 2.24 & 227 & 11.01 & 0.000 \\
\hline Casi nunca & 797 & 2.82 & 89 & $|4.6|$ & 0.000 \\
\hline \multicolumn{6}{|c|}{ Satisfecho con el tiempo de convivencia familiar } \\
\hline Casi siempre & 2121 & 1.60 & 226 & 10.62 & 0.000 \\
\hline A veces & 1464 & 2.19 & 242 & 11.16 & 0.000 \\
\hline Casi nunca & 490 & 3.47 & 82 & 20.73 & 0.000 \\
\hline \multicolumn{6}{|c|}{ ¿Sientes que tu familia te quiere? } \\
\hline Casi siempre & 2820 & 1.63 & 342 & 9.36 & 0.000 \\
\hline A veces & 1030 & 2.52 & 227 & 15.12 & 0.000 \\
\hline Casi nunca & 225 & 4.89 & 93 & 27.78 & 0.000 \\
\hline
\end{tabular}

\footnotetext{
* Antecedente de consumo de drogas ilegales

‡ Valor "p" de la diferencia de prevalencias
} 


\section{Cuadro III}

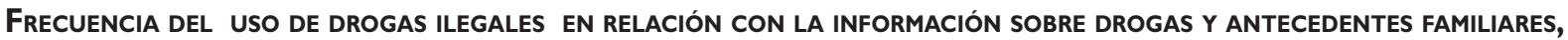
estratificado por antecedente de tabaquismo, Morelos, México, 2005

\begin{tabular}{|c|c|c|c|c|c|}
\hline \multirow[b]{2}{*}{ Variable } & \multicolumn{2}{|c|}{ No fumadores } & \multicolumn{3}{|c|}{ Fumadores } \\
\hline & $n$ & $\%^{*}$ & $n$ & $\%^{*}$ & Valor " $p$ " \\
\hline \multicolumn{6}{|c|}{ Consumo de drogas por los padres } \\
\hline Ninguno & 4007 & 1.90 & 534 & 12.17 & 0.000 \\
\hline Alguno & 68 & 10.29 & 16 & 18.75 & 0.347 \\
\hline \multicolumn{6}{|c|}{ Consumo de drogas por amigos } \\
\hline No & 3776 & 1.56 & 440 & 8.64 & 0.000 \\
\hline Sí & 299 & 8.03 & 28 & 27.27 & 0.001 \\
\hline \multicolumn{6}{|c|}{ Información de las drogas por parte de los padres } \\
\hline Sí, siempre & 2124 & 2.07 & 304 & 10.20 & 0.000 \\
\hline Sí, algunas veces & 1630 & 1.53 & 201 & 15.42 & 0.000 \\
\hline Casi nunca & 130 & 2.31 & 22 & 22.73 & 0.000 \\
\hline Nunca & 191 & 5.76 & 23 & 4.35 & $0.78 \mathrm{I}$ \\
\hline \multicolumn{6}{|c|}{ Información de las drogas por parte de los profesores } \\
\hline Sí, siempre & 116 & 2.15 & 186 & 12.37 & 0.002 \\
\hline Sí, algunas veces & 2410 & 1.83 & 279 & 12.90 & 0.000 \\
\hline Casi nunca & 284 & 0.55 & 51 & 4.76 & 0.010 \\
\hline Nunca & 265 & 5.28 & 34 & $|4.7|$ & 0.033 \\
\hline \multicolumn{6}{|c|}{ ¿Te han ofrecido drogas en la casa? } \\
\hline No & 4007 & 1.90 & 529 & 11.72 & 0.000 \\
\hline Sí & 68 & 10.29 & 21 & 28.57 & 0.038 \\
\hline \multicolumn{6}{|c|}{ ¿Te han ofrecido drogas en la escuela? } \\
\hline No & 3965 & 1.97 & 519 & 11.95 & 0.000 \\
\hline Sí & 110 & 4.55 & 31 & 19.35 & 0.007 \\
\hline \multicolumn{6}{|c|}{ ¿Te han ofrecido drogas en fiestas? } \\
\hline No & 3958 & 1.92 & 476 & 8.82 & 0.000 \\
\hline Sí & 117 & 5.98 & 74 & 35.14 & 0.000 \\
\hline \multicolumn{6}{|c|}{ ¿Te han ofrecido drogas en discotecas? } \\
\hline No & 4066 & 2.02 & 542 & 12.36 & 0.000 \\
\hline Sí & 9 & II.II & 8 & 12.50 & 0.929 \\
\hline \multicolumn{6}{|c|}{ ¿Te han ofrecido drogas en la calle? } \\
\hline No & 3850 & 1.90 & 454 & 10.57 & 0.000 \\
\hline Sí & 225 & 4.44 & 96 & 20.83 & 0.000 \\
\hline \multicolumn{6}{|c|}{ * Antecedente de consumo de drogas ilegales } \\
\hline ‡ Valor " $p$ " de la dife & & & & & \\
\hline
\end{tabular}

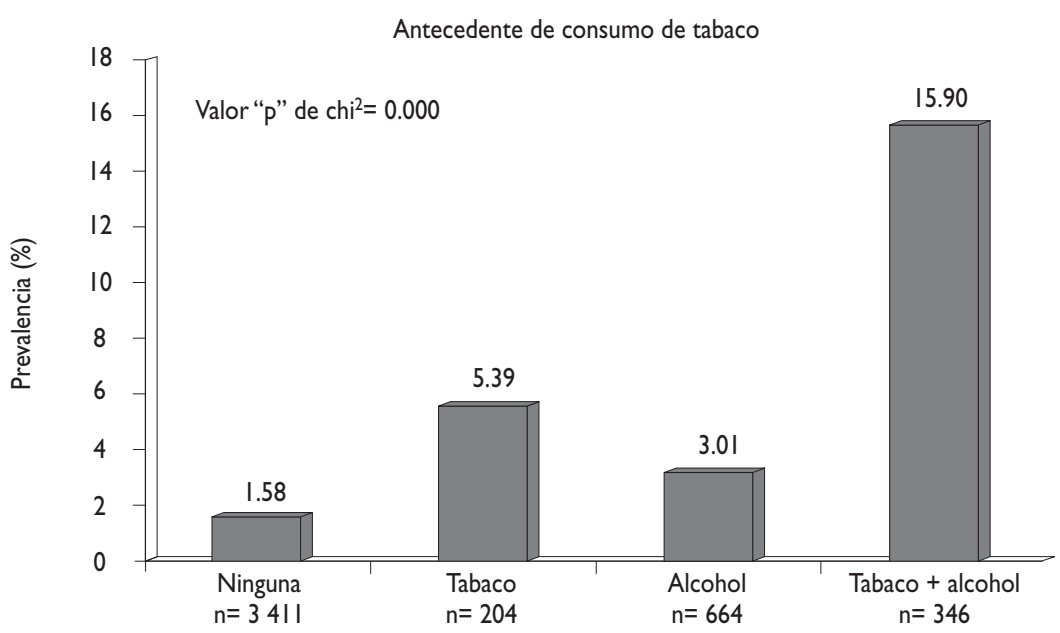

Figura 2. Distribución del CONSUMO de dROGAS ILEgALES DE ACUERDO AL ANTECEDENTE DE CONSUMO de ALCOHOL Y TABACO en estudiantes de Morelos, México, 2005 


\section{Cuadro IV}

Determinantes del uso de dROgas ILEgales en función del CONSUMO de tabaco en estudiantes del estadio de Morelos, México, 2005

\begin{tabular}{|c|c|c|c|c|c|c|}
\hline \multirow{3}{*}{ Género $\quad$ Variable } & \multicolumn{3}{|c|}{ No fumadores } & \multicolumn{3}{|c|}{ Antecedente de fumar } \\
\hline & \multirow[t]{2}{*}{$O R^{*}$} & \multicolumn{2}{|c|}{$I C 95 \%$} & \multirow[t]{2}{*}{$O R^{*}$} & \multicolumn{2}{|c|}{$1 C 95 \%$} \\
\hline & & & & & & \\
\hline Mujeres & 1.00 & & & 1.00 & & \\
\hline Hombres & 0.80 & 0.45 & I.4I & 0.59 & 0.25 & I.4I \\
\hline \multicolumn{7}{|l|}{ Escolaridad } \\
\hline Secundaria & 2.67 & 1.08 & 6.60 & 1.97 & 0.74 & 5.25 \\
\hline Preparatoria/Universidad & 1.00 & & & 1.00 & & \\
\hline \multicolumn{7}{|l|}{ Ingreso familiar } \\
\hline$\leq \$ 600.00$ & 1.00 & & & 1.00 & & \\
\hline$\$ 600.00$ a $\$ 1,200.00$ & 0.68 & 0.38 & 1.20 & 1.60 & 0.75 & 3.39 \\
\hline$\$ 1,201.00$ a $\$ 2,400.00$ & 0.77 & 0.39 & $\mathrm{I} .54$ & 1.19 & 0.48 & 2.96 \\
\hline$>\$ 2,400.00$ & 0.77 & 0.38 & 1.57 & 1.02 & 0.32 & 3.17 \\
\hline \multicolumn{7}{|l|}{ Sintomatología depresiva } \\
\hline Sin síntomas & 1.00 & & & 1.00 & & \\
\hline Con síntomas & 0.81 & 0.45 & 1.48 & 1.17 & 0.53 & 2.54 \\
\hline \multicolumn{7}{|l|}{ Estado civil de los padres } \\
\hline Con pareja (casados, unión libre) & 1.00 & & & 1.00 & & \\
\hline Separados (divorciados, viudos, madres solteras, separados) & 1.53 & 0.90 & 2.58 & 1.02 & 0.54 & 1.89 \\
\hline \multicolumn{7}{|l|}{ Consumo de drogas por los padres } \\
\hline Ninguno & 1.00 & & & 1.00 & & \\
\hline Alguno & 4.54 & 1.87 & 11.02 & 2.04 & 0.49 & 8.38 \\
\hline \multicolumn{7}{|l|}{ Antecedente de consumo de alcohol alguna vez en la vida } \\
\hline No & 1.00 & & & 1.00 & & \\
\hline Sí & 1.77 & 0.95 & 3.29 & 2.68 & 1.21 & 5.91 \\
\hline \multicolumn{7}{|l|}{ Consumo de drogas por amigos } \\
\hline No & 1.00 & & & 1.00 & & \\
\hline Sí & 5.49 & 2.90 & 10.42 & 4.52 & 1.86 & 11.00 \\
\hline \multicolumn{7}{|l|}{ Información de las drogas por parte de los padres } \\
\hline Sí, siempre & 1.00 & & & 1.00 & & \\
\hline Sí, algunas veces & 0.64 & 0.38 & 1.07 & 1.49 & 0.81 & 2.75 \\
\hline Casi nunca & 0.93 & 0.27 & 3.15 & 2.28 & 0.66 & 7.78 \\
\hline Nunca & 1.72 & 0.81 & 3.62 & 0.40 & 0.04 & 3.50 \\
\hline \multicolumn{7}{|l|}{ ¿Sientes que tu familia te quiere? } \\
\hline Casi siempre & 1.00 & & & 1.00 & & \\
\hline A veces & 1.15 & 0.68 & 1.94 & 1.69 & 0.89 & 3.19 \\
\hline Casi nunca & 1.78 & 0.85 & 3.73 & 4.61 & 1.76 & 12.07 \\
\hline \multicolumn{7}{|l|}{ ¿Te han ofrecido drogas en tu casa? } \\
\hline No & 1.00 & & & 1.00 & & \\
\hline Sí & 5.33 & 2.18 & 13.04 & 3.89 & 1.22 & 12.38 \\
\hline \multicolumn{7}{|l|}{ ¿Te han ofrecido drogas en fiestas? } \\
\hline No & 1.00 & & & 1.00 & & \\
\hline Sí & 1.81 & 0.76 & 1.26 & 3.92 & 1.95 & 7.86 \\
\hline
\end{tabular}

* OR ajustado por todas las variables del cuadro, edad y el índice de propensidad de la variable de antecedente de consumo de tabaco.

contestaron "casi siempre", tiene una mayor posibilidad $(O R=4.6 ;$ IC95\% 1.7-12.0). Respecto a los lugares donde les han ofrecido drogas ilegales, para ambos estratos de antecedente de consumo de tabaco el hecho de que alguien perteneciente al hogar les ofrezca drogas ilegales se asocia con el uso de éstas (no fumadores: $O R=5.3$; IC95\% 2.1-13.0; fumadores: $O R=3.8$; IC95\% 1.2-12.3). El ofrecimiento de drogas ilegales en fiestas mostró relación sólo con aquellos que tienen el antecedente de fumar. La prueba de bondad de ajuste de HosmerLemeshow para el modelo de los no fumadores fue de $4.87, p=0.771$; y para el modelo de los fumadores fue de $4.86, p=0.769$.

\section{Discusión}

En el contexto de la población mexicana, los resultados del presente estudio plantean el problema complejo del uso de sustancias adictivas en los jóvenes. El consumo 
de tabaco y otras drogas es una conducta que manifiesta la necesidad de los individuos de utilizar sustancias psicoactivas para enfrentar los problemas cotidianos. Por lo tanto, es importante el abordaje del estudio conjunto del consumo del tabaco y otras drogas pues determina los diferentes perfiles de población respecto del uso de sustancias adictivas y su interacción.

Entre los resultados de este trabajo se observa que, en relación con el género, el uso de drogas ilegales es similar entre hombres y mujeres independientemente del antecedente de fumar o no. Empero, para aquellos que no consumen tabaco, la exposición al antecedente familiar y de pares de uso de drogas ilegales ejerce un efecto mucho mayor que en los jóvenes que consumen tabaco, lo que podr a explicar que para este grupo no haya un determinante fisiológico que facilite el uso de drogas, sino que más bien se trate de la accesibilidad de los jóvenes a las drogas ilegales a través de su oferta por parte de personas y ambientes cercanos a ellos. Lo anterior coincide con lo informado en otros estudios, donde se muestra que los mecanismos de exposición iniciales y más frecuentes son el ofrecimiento de la droga como regalo hecho por algún amigo, compañero o conocido, o bien la invitación a usarla por alguien que intenta venderla. ${ }^{25}$ En contraparte, Rojas-Guiot y colaboradores ${ }^{26}$ consideran que la percepción de un ambiente familiar funcional es una caracter stica que puede impedir que los jóvenes consuman drogas o alcohol. Lo contrario sucede al existir una percepción de apoyos y controles familiares débiles, que puede predecir el uso de drogas entre estudiantes adolescentes. Lo cierto es que la mayor a de los padres de jóvenes que se encuentran en esta etapa de la vida tiene la expectativa de que sus hijos no fumen, pero también es cierto que puede resultarles dif cil encontrar los mejores mecanismos para comunicar sus expectativas respecto al consumo de tabaco. ${ }^{27}$

De acuerdo con el tipo de droga utilizada, este estudio mostró que aquellos estudiantes que ten an antecedente de fumar informaron un mayor uso de coca na y marihuana, datos que coinciden con lo notificado en otros estudios. ${ }^{28}$ En contraste, en los resultados se observa que en los jóvenes con antecedente de fumar no hay un efecto del uso de drogas ilegales por parte de los padres, y que el uso de drogas por parte de los amigos tiene una menor fuerza de asociación; sin embargo, el efecto del consumo de alcohol se relaciona como factor que explica el uso de drogas ilegales entre los jóvenes.

De manera previa a este estudio, en la población de estudiantes de Morelos encuestados en 1998, se observó que la relación de consumo de tabaco o alcohol, junto con la variable de género, modifica el efecto sobre el uso de drogas ilegales. Se hizo evidente que los hombres que consum an tabaco o alcohol tuvieron 9.2 veces más (IC95\% 5.2-16.3) la posibilidad de consumir drogas ilegales con respecto a aquellos que no consum an tabaco o alcohol, mientras que las mujeres que fumaban o consum an alcohol tuvieron menor riesgo de consumir drogas ilegales $(R R=3.4$; IC95\% 1.9-6.4), resultados que ubican al consumo de alcohol o tabaco como un importante antecedente del inicio en el consumo de otras sustancias ya que se observa que la proporción de usuarios aumenta con la edad y documenta, una vez más, un exceso de riesgo asociado al sexo masculino. ${ }^{29}$

El presente estudio difiere del anterior respecto al uso de sustancias ilegales de acuerdo con el género del estudiante y el antecedente de fumar, en donde se destaca que no existen diferencias genéricas en el uso de drogas ilegales entre quienes consumen tabaco. Cabe mencionar que para el presente estudio se definió como fumador al que ten a antecedente de fumar antes o actualmente, sin considerar el tiempo que lleve haciéndolo. No obstante, la variable de consumo de alcohol dentro del estrato de los fumadores s mostró una relación de mayor posibilidad en relación con el uso de drogas ilegales. En un estudio realizado en escolares de 14-18 años de edad, en Hungr a, ${ }^{30}$ se observó que 20\% del total de alumnos hab a tenido alguna experiencia con una droga. La definición de uso de drogas en el presente trabajo es de "aquellos que hayan usado drogas alguna vez en la vida". En el estudio de Hungr a, los varones tuvieron una mayor prevalencia de consumo que las mujeres $\left(X^{2}=4.65 ; p=0.031\right)$; la marihuana fue la droga de consumo más común $(96 \%)$ y la proporción de usuarios de drogas fue significativamente mayor en aquellos que hab an fumado (13\%) o en los que fumaban entonces $(65 \%)$, comparados con aquellos que nunca hab an fumado $\left(1 \% ; X^{2}=164.7 ; p<0.001\right)$. La posible hipótesis ser a que la necesidad f sica de consumir drogas ilegales tiene un mayor peso en la población que también ha estado expuesta al tabaco y al alcohol, conductas que se relacionan con caracter sticas psicológicas y emocionales de los individuos, y que en parte fueron evaluadas por el ndice de depresión, aunque éste no fue estad sticamente significativo debido, tal vez, a una falta de poder.

La relación fisiológica entre el consumo de tabaco y otras drogas puede explicarse de la siguiente manera: el tabaquismo ejerce un efecto drástico en el cerebro, ya que se ha encontrado una disminución marcada en los valores de la enzima monoaminooxidasa (MAO), que metaboliza a la dopamina. La disminución en las dos formas de la MAO (A y B) determina concentraciones más altas de dopamina, que a su vez dan lugar al deseo de usar la droga en forma repetida, lo cual podr a explicar la compulsión de los individuos por fumar. ${ }^{31}$ 
Se puede observar el mismo efecto con el consumo de alcohol, ya que en estudios llevados a cabo en animales y humanos se apreció que la administración previa de alcohol incrementa la posibilidad de fumar. ${ }^{32}$ Esta asociación puede explicarse porque ambas sustancias (el etanol y la nicotina) estimulan los mismos receptores dopaminérgicos y, de esta manera, originan el deseo compulsivo de una sustancia por la otra, ${ }^{33}$ efecto similar al que presentan otras drogas como la coca na. ${ }^{33,35} \mathrm{~A} \mathrm{su}$ vez, se ha postulado la hipótesis de que los aspectos genéticos pueden intervenir en la susceptibilidad de los individuos a la estimulación de las sustancias adictivas pues se ha observado que la variación del gen DAT1 puede desempeñar un papel en la etiolog a de la dependencia a la coca na. ${ }^{36}$

En el caso de los adolescentes no fumadores, por ejemplo, existe una determinante que ha resultado significativa en el consumo futuro de tabaco conocida como susceptibilidad y que incluye intenciones y expectativas de conductas futuras. Se trata de un mecanismo que consiste en identificar a aquellas personas con una predisposición cognitiva para fumar e incluye aspectos como: actitudes basadas en creencias favorables y desfavorables sobre el consumo de tabaco, autoeficacia para resistir las presiones de los pares, percepción de riesgo, búsqueda de sensaciones como un rasgo de personalidad, influencia de modelos sociales fumadores, etcétera. ${ }^{37,38}$

Lamentablemente, existe evidencia de que la pertenencia a grupos de pares que fuman tiene una elevada probabilidad de que los jóvenes experimenten el tabaco y que continúen con la iniciación de su consumo. ${ }^{39}$ En el caso del uso de drogas ilegales, es probable que se trate de un mecanismo similar al propuesto en el caso del tabaco. Los adolescentes pueden mostrar una tendencia a aceptar e iniciar el uso de drogas de acuerdo con el entorno familiar y de pares, que ejercen un efecto de presión para este grupo de adolescentes. Lo cierto es que se necesita realizar estudios de seguimiento que permitan evaluar el efecto conjunto del uso de drogas ilegales en aquellos jóvenes que nunca han fumado. Por otro lado, también se requiere evaluar la autoeficacia o aquellas situaciones que le permiten al adolescente contrarrestar el efecto de la familia, en particular, en el consumo de drogas, sobre todo cuando quien lo hace es alguno de los padres, as como de los pares.

Respecto a otras variables de ndole emocional, como las relacionadas con su percepción de la relación familiar, se observa que la percepción de ausencia de afecto por parte de sus familiares en los jóvenes se asoció positivamente con la posibilidad de consumir drogas ilegales, en especial en aquellos que exhiben el antecedente de fumar, situación que no se observa en los que no lo tienen. Estudios previos evidencian el papel del funcionamiento familiar y el uso de sustancias en adolescentes, en conjunto con su autoestima. Cabe mencionar que las caracter sticas del funcionamiento familiar sólo constituyen un factor de explicación distal del uso de sustancias en adolescentes; en otras palabras, las caracter sticas positivas o negativas del funcionamiento familiar potencian o inhiben las autoevaluaciones positivas del adolescente en los distintos dominios de su vida, autoevaluaciones que son, a su vez, importantes predictores del uso de sustancias en los adolescentes. ${ }^{35,40}$

La presencia de s ntomas depresivos fue relativamente poco asociada, as como la autoeficacia no se relacionó con el consumo de drogas ilegales en este estudio. ${ }^{41}$ Estos resultados están en 1 nea con los de otros trabajos, donde no se observa que los trastornos internos guarden una relación significativa con la posibilidad del uso de sustancias, excepto en el caso de depresión mayor. ${ }^{42}$

Otro resultado importante de este estudio se observa en el grado educativo al que pertenec an los participantes, sobre todo en los jóvenes que carecen del antecedente de fumar, ya que la posibilidad de consumir drogas ilegales es mayor en aquellos que cursan la secundaria en comparación con los de preparatoria y profesional. Lo anterior puede explicarse por el hecho de que si bien el consumo de tabaco es menor en este grupo debido a las medidas de prevención y control que se implantaron en México a partir de 2004, ${ }^{43}$ también los más jóvenes de la población de estudio tienen mayor acceso a las drogas ilegales, lo que representa un alerta para la prevención y control de las adicciones, y el consumo de tabaco, alcohol y drogas ilegales en el pa s.

\section{Limitaciones}

El diseño original del estudio fue de cohorte, pero se analizó únicamente la medición basal y por consecuencia, los resultados de este trabajo no establecen relaciones causales debido a la falta de temporalidad de la asociación exposición-efecto. ${ }^{44}$ Asimismo, se considera que los datos obtenidos mediante un autoinforme pueden subestimar el fenómeno en estudio, es decir que la prevalencia real podr a ser más alta que la notificada; $\sin$ embargo, está documentada la validez de los cuestionarios autoaplicables para la medición del uso de drogas il citas, alcohol y tabaco. ${ }^{2,45}$ Los resultados sólo pueden extrapolarse a estudiantes de escuelas públicas y con caracter sticas similares a las de la muestra.

\section{Conclusiones}

Se requieren propuestas de pol ticas para reducir, o cesar, el consumo de sustancias adictivas a partir de un enfoque integral que incluya al tabaco, al alcohol y a las 
drogas ilegales en adolescentes a través de intervenciones preventivas. Son recomendables los planes de acción en las escuelas, en particular los dirigidos a los padres de familia, en el interés de proveer información sobre los riesgos a la salud que ocasiona el consumo de estas sustancias, como son el cáncer pulmonar, gástrico y de la piel, entre otros, en el caso del tabaco, y las afecciones hepáticas y biliares debidas a la ingesta inmoderada de alcohol, además de los efectos directos sobre el sistema nervioso que ocasiona el consumo de drogas ilegales. Asimismo, es indispensable mantener mecanismos de comunicación adecuados con los adolescentes respecto de variables como la autoestima y la depresión que favorezcan una vida donde priven la armon a y la salud mental.

Los profesionales de la educación en los sectores de la salud tendr an que sensibilizarse respecto de estos tópicos, y considerar en especial algunos factores que se relacionan con la composición familiar (vivir con ambos padres o con alguno de ellos), la vigilancia de las actividades de los adolescentes, el consumo de sustancias adictivas, de tabaco y alcohol por parte de otros familiares y/o amigos, el desempeño escolar as como el comportamiento del joven en otros entornos sociales que le impidan lograr un desarrollo f sico y mental armónico. Como actividad inicial, para la realización de todo lo anterior, ser a recomendable establecer un sistema de vigilancia epidemiológica en el contexto de la educación secundaria en escuelas públicas, en el que con cada ingreso al año escolar se aplique una encuesta sobre consumo de sustancias adictivas (tabaco, alcohol, drogas ilegales), al igual que se midan otros factores de riesgo, como actividad f sica, obesidad, etc. El objetivo ser a llevar un seguimiento y poder establecer indicadores que permitan evaluar los programas preventivos que se implementen en las escuelas.

\section{Agradecimientos}

Esta investigación se llevó a cabo gracias al financiamiento SALUD-2003-C01-059 aportado por el Consejo Nacional de Ciencia y Tecnolog a (CONACYT) de México.

\section{Referencias}

I. Adlaf EM, Paglia A, Ivis FJ, lalomiteanu A. Nonmedical drug use among adolescent students: Highlights from the 1999 Ontario Student Drug Use Survey. CMAJ 2000; 162:1677-1680.

2. Bauman A, Phongsavan P. Epidemiology of substance use in adolescence: Prevalence, trends and policy implications. Drug Alcohol Depend
1999;55:187-207.

3. Sutherland I, Shepherd JP.The prevalence of alcohol, cigarette and illicit drug use in a stratified sample of English adolescents. Addiction 200I;96:637-640.

4. Smart RG, Ogborne AC. Drug use and drinking among students in 36 countries. Addict Behav 2000;25:455-460.

5. Mendoza-Berjano R, Batista-Foguet JM, Sánchez-García M, CarrascoGonzález AM. The consumption of tobacco, alcohol and other drugs by adolescent Spanish students. Gac Sanit 1998; I2:263-27I.

6. Rosovsky H, Cravioto-Quintana P, Medina-Mora ME. El consumo de drogas en México: diagnóstico, tendencias y acciones. México, D. F: Secretaría de Salud/Consejo Nacional contra las Adicciones, 1999:87-100. 7. Arillo-Santillán E, Lazcano-Ponce E, Sánchez-Zamorano LM,Valdés $R$, Fernández E, Samet JM, et al. Estudio poblacional de tabaquismo en adolescentes de Morelos. Factores asociados a la prevalencia, incidencia, persistencia y cesación, 1998-2003. En: Primer informe sobre el combate al tabaquismo. México ante el Convenio Marco Para el Control del Tabaco. Cuernavaca, México: Instituto Nacional de Salud Pública 2005:47-64.

8. Consejo Nacional Contra las Adicciones. Encuesta Nacional de Adicciones 2002: tabaco, alcohol y otras drogas, resumen ejecutivo. México, D. F.: Secretaría de Salud, 2003:9-14.

9.Valdés-Salgado R, Thrasher J, Sánchez-Zamorano LM, Lazcano-Ponce E, Reynales-Shigematsu LM, Meneses González F et al. Los retos del convenio marco para el control del tabaco en México: un diagnóstico a partir de la Encuesta sobre Tabaquismo en Jóvenes. Salud Publica Mex 2006;48 Supp I:S5-SI6.

10. Olaiz-Fernández G, Rivera-Dommarco J, Shamah-Levy T, Rojas R, Villalpando-Hernández S, Hernández-Avila M, et al. Encuesta Nacional de Salud y Nutrición 2006. Cuernavaca, México: Instituto Nacional de Salud Pública 2006:67-74.

II. Kandel DB. Stages in adolescent involvement in drug use. Science 1975;190:912-914.

12. Kandel DB, Yamaguchi K, Chen K. Stages of progression in drug involvement from adolescence to adulthood: Further evidence for the gateway theory. J Stud Alcohol 1992;53:447-457.

13. Robins LN. Estimating addiction rates and locating target populations: How decomposition into stages helps. En: Rittenhouse JD, ed. The epidemiology of heroin and other narcotics. NIDA Research Monograph 16. Rockville (MD): National Institute on Drug Abuse; 1977:25-39.

I4. Willner P.A view through the gateway: Expectancies as a possible pathway from alcohol to cannabis.Addiction 2001;96:691-703.

I5. Neumark YD, Delva J,Anthony JC. The epidemiology of adolescent inhalant drug involvement. Arch Pediatr Adolesc Med 1998; 152:781-786. 16. Jessor R, Donovan JE,Widner K. Psychosocial factors in alcohol and drug use. The 1978 National Sample Study of the 1974-78 panel study. Boulder, Col. Boulder Institute of Behavioral Science, University of Colorado, 1980.

17. Secretaría de Educación Pública, Instituto Mexicano de Psiquiatría. Encuesta Nacional sobre el Uso de Drogas entre la Comunidad Escolar Nacional. México, D. F.: SEP, IMP; 1993.

18. Soto F. El desarrollo de la adolescencia. FD Órgano Informativo de los Centros de Integración Juvenil 1982;3:22-26.

19. Mac Nally MT, Menéndez I, Rabetzky N,Viale C. Etnografía de la droga: valores y creencias en los adolescentes. Rev Esp Antropol Am 1993; VIII:I3-25.

20. Maier H.Tres teorías sobre el desarrollo del niño: Erikson, Piaget y Sears. Buenos Aires:Amorrortu Editores, 1982.

21. Castro ME, Llanes J. La prevención de los riesgos psicosociales en la vida cotidiana. CONADIC informa 2002:24-28.

22. Zung WWK.A self rating depression scale. Arch Gen Psychiatry 1965; I2:63-70. 
23. Seva-Diaz A. La depresión y su evaluación. En: Rabasdsa B (ed). Estudio sociológico "libro blanco" de la depresión en España. Madrid: Gabinete de estudios sociológicos Bernard Krief Lederle Eds., 1982:75-84.

24. Stata Corporation, 4905 Lakeway Drive Special Edition College Station, Texas 77845 USA 800-STATA-PC

25. Wagner FA, González-Forteza C, Aguilera RM, Ramos-Lira LE, MedinaMora ME,Anthony JC. Oportunidades de exposición al uso de drogas entre estudiantes de secundaria de la Ciudad de México. Salud Mental 2003;26:22-32.

26. Rojas-Guiot E, Fleiz-Bautista C, Medina-Mora Icaza ME, Morón MA, Doménech-Rodríguez M. Consumo de alcohol y drogas en estudiantes de Pachuca, Hidalgo. Salud Publica Mex 1999;41:297-308.

27. Simons-Morton BG, Haynie DL. Psychosocial predictors of increased smoking stage among sixth graders. Am J Health Behav 2003;27:592-602. 28. Barrett SP, Darredeau C, Pihl RO. Patterns of simultaneous polysubstance use in drug using university students. Hum Psychopharmacol 2006; 21:255-263.

29. Herrera-Vázquez M,Wagner FA, Velasco-Mondragón E, Borges G Lazcano-Ponce E. Inicio en el consumo de alcohol y tabaco y transición a otras drogas en estudiantes de Morelos, México. Salud Publica Mex 2004:46: I32-140.

30. Nyari TA, Heredi K, Parker L.Addictive behavior of adolescents in secondary schools in Hungary. Eur Addict Res 2005; I :38-43

3I. NIDA Serie de reportes de Investigación - Adicción a la Nicotina: NIH Publicación Número 01-4342, abril 2002.

32. Shiffman S, Balabanis M.Associations between alcohol and tobacco. En: Fertig JB,Allen JP eds.Alcohol and tobacco: from basic science to clinical practice. NIAAA Research Monograph No. 30. Bethesda, MD: US Department of Health and Human Services, 1995: 17-36.

33. Wise RA. The neurobiology of craving: implications for the understanding and treatment of addiction. J Abnorm Psycol 1988; 97:1 18-132.
34. Badanich KA,Adler KJ, Kirstein CL.Adolescents differ from adults in cocaine conditioned place preference and cocaine-induced dopamine in the nucleus accumbens septi. Eur J Pharmacol 2006; 550:95-106.

35. Ridenoura TA, Lanzaa ST, Donnyb EC, Clarkb DB. Different lengths of times for progressions in adolescent substance involvement. Addict Behav 2006;31:962-983.

36. Guindalini C, Howard M, Haddley K, Laranjeira R, Collier D, Ammar $\mathrm{N}$ et al.A dopamine transporter gene functional variant associated with cocaine abuse in a Brazilian sample. PNAS 2006; 103:4552-4557.

37. Simons-Morton BG. The protective effect of parental expectations against adolescent smoking initiation. Health Educ Res 2004;19:56I-569. 38. Choi WS, Gilpin EA, Farkas AJ, Pierce JP. Determining the probability of future smoking among adolescents. Addiction 200I;96:3।3-323.

39. Conrad KM, Flay BR, Hill D.Why children start smoking cigarettes: predictors of onset. J Addiction 1992;87: I7 I I-I724.

40. Musito G, Jiménez TI, Murgui S. Funcionamiento familiar, autoestima y consumo de sustancias en adolescentes: un modelo de mediación. Salud Publica Mex 2007;49:3-10.

4I. Roberts RE, Ramsay-Roberts C, Xing Y. Comorbidity of substance use disorders and other psychiatric disorders among adolescents: Evidence from an epidemiologic survey. Drug Alcohol Depend 2007;88 Suppl: S4-SI3.

42. King SM, lacono WG, McGue M. Childhood externalizing and internalizing psychopathology in the prediction of early substance use. Addiction 2004;99:1548-1559.

43. Secretaría de Salud. Decreto de reforma y adición de diversas disposiciones de la Ley General de Salud en relación a la publicidad del tabaco. Diario Oficial, lunes 19 de enero de 2004:40-4I.

44. Hernández B,Velasco-Mondragón HE. Encuestas transversales. Salud Publica Mex 2000;42:447-455.

45. Goodstadt MS, Chan GC, Sheppard MA, Cleve JC. Consistencies in self-reported drug use among students in Ontario (Canada). Bull Narc 1985;37:55-65. 